\title{
Clinical Impact of Preoperative Neutrophil-Lymphocyte Ratio in Renal Cell Carcinoma in Sardjito Hospital
}

\author{
Bagus Gilang Samudra ${ }^{1}$, Aria Danurdoro ${ }^{1 *}$ Danarto $^{2}$ \\ ${ }^{1}$ Faculty of Medicine, Gadjah Mada University, Dr. Sardjito Hospital, Yogyakarta, Indonesia \\ 2 Urology Division, Department of Surgery, Faculty of Medicine, Gadjah Mada University, Dr. Sardjito Hospital, \\ Yogyakarta, Indonesia
}

\section{ARTICLE INFO}

Received : 30 September 2020

Reviewed : 25 November 2020

Accepted : 23 December 2020

\section{Keywords:}

neutrophil-lymphocyte ratio,

renal cell carcinoma
*Corresponding author:

\section{Aria Danurdoro}

Division of Urology, Department

of Surgery, Faculty of Medicine,

Public Health and Nursing, Universitas

Gadjah Mada - Dr. Sardjito Hospital

danurdoroaria@gmail.com

\section{A BSTRACT}

Background: Renal cell carcinoma (RCC) is one of the most common malignancies in the kidney and causes a mortality rate of more than 100,000 each year globally. The neutrophil-lymphocyte ratio (NLR) is one of the markers in the inflammatory response that also correlate with the alteration of any cancer cells. We investigated the correlation between the NLR on tumor stage, Fuhrman nuclear grade, length of stay (LOS), mortality, and recurrence rate in renal cell carcinoma.

Methods: We investigated 52 patients with renal cell carcinoma retrospectively from the databases of the patients who underwent radical and partial nephrectomy in Sardjito Hospital Yogyakarta between 2012 and 2017. Patients were classified as lower stage (T1-T2) and higher stage (T3-T4). We also classified it as a group of Fuhrman nuclear grade (G1, G2, G3, and G4). Two sample t-test or one-way ANOVA was used for the continuous variables and a chi-square test or Fisher's exact test for the categorical variables. Pearson test was used to do the correlation test.

Results: Among a total of 52 patients analyzed in our study, there were 36 males (69\%) and 16 females (31\%). A normal distribution of datasets was verified. The mean of preoperative NLR in these 52 patients was $6.35(+4.01)$, with a range of $1.48-21.0$. The value of NLR was positively correlated with tumor pathologic stage $(p=0.020)$, length of stay of the patients $(p=0.00)$, and the mortality rate $(p=0.012)$. Neutrophil lymphocyte ratio was not significantly correlated with Fuhrman tumor grade $(p=0.357)$ and tumor recurrence $(p=0.670)$.

Conclusions: We compared the relationship of preoperative NLR with renal cell carcinoma tumor stage $(T)$, Fuhrman grade, length of stay, mortality, and tumor recurrence. NLR was found to have a statistically significant higher $\mathrm{T}$ stage, mortality, and length of stay of the patients. Further studies with more patients are needed to confirm our study.

\section{INTRODUCTION}

Renal cell carcinoma (RCC) is the most common type of renal malignancy in adult kidneys and causes widespread mortality of over 100,000 per year [1,2]. In the United States, RCC causes approximately $2.3 \%$ of all cancer deaths [3]. In the United States, the estimated numbers of new cases and deaths in 2015 were 61,500 and 14,080 [2]. Moreover, it is important to optimize decision-making on the treatment and prognosis of RCC and to provide better patient counseling. Until now, many of the characteristics of
RCC alone have been suggested as prognostic factors [4]. However, only a few of these factors include the pathological grade and Fuhrman grade for prognostic factors for RCC.

The NLR (neutrophil-to-lymphocyte ratio) is a widely used marker of inflammation to assess the outcome in critically ill surgical patients and provides a good indication of the patient's overall inflammatory status. Increased NLR is an indicator for poor outcome in cardiopathic and vasculopathy patients undergoing intervention and is also a predictor of poor survival in cancer patients [5]. 
The management approach to RCC is guided by the probability of cure, which is related directly to the stage or degree of tumor dissemination. A survey from the American Cancer Society revealed differences of survival kidney cancer according to tumor stages [6]. This warrants early detection or prognostic indicators for RCC because it is essential for early diagnosis and to aid in the management of patients. NLR is an easily measured and highly reproducible test to be incorporated in daily practice pre-operatively in many malignancies; determined by dividing the number of neutrophils by the number of lymphocytes, NLR is widely considered to be the potential surrogate of systemic inflammatory status and adaptive immunity balances. The significance of NLR and its correlation to RCC is still questionable, especially in the Indonesian population. In this study, we evaluated the correlation of the preoperative NLR on tumor stage, Fuhrman nuclear grade, length of stay, mortality, and recurrence in renal cell carcinoma.

\section{METHODS}

Medical records of 52 patients newly diagnosed and undergoing surgery for RCC between 2012 and 2017 in Sardjito General Hospital were retrospectively collected and analyzed. The enrolled patients met the following inclusion criteria: (1) all patients were diagnosed histopathologically with RCC and underwent radical or partial nephrectomy for a primary lesion; (2) no hematology disease, infection, hyperpyrexia, and pathologic condition/injury of the kidney; (3) inflammatory markers were obtained prior to anticancer treatment, such as surgery, blood transfusion, chemotherapy, and radiotherapy; (4) informed consents were obtained from eligible patients. No patients were excluded due to eligibility criteria. NLR was defined as the neutrophil counts divided by the lymphocyte counts. This inflammatory marker was calculated from the presurgical blood sample analysis, measuring the white cell, neutrophil, lymphocyte, and platelet counts. We observed the preoperative NLR and compared it with the renal cell carcinoma tumor stage $(T)$, Fuhrman grade, length of stay, mortality, and tumor recurrence. The diagnosis of RCC was made depending on histological evidence and classified based on the eighth edition of the TNM-UICC/AJCC classification [7]. The staging was performed preoperatively with CT scan and was confirmed on the histopathology report established by certified pathologists after surgery. The RCC recurrence, either local or distant, deemed by the treating surgeon, was described by the appearance of tumor enhancement on contrast-enhanced CT imaging or magnetic resonance imaging (MRI). This could be present at the same or another portion of the kidney or even metastasis to other organ systems.
Statistical analysis. Patient baseline features were summarized using descriptive statistics. For quantitative variables, two-sample t-test and one-way ANOVA were performed to derive the $p$-value. Associations between two categorical characteristics were examined by Pearson's Chi-square test. All data were analyzed using the SPSS v23 software (SPSS/IBM, Chicago, IL). A p-value $<0.05$ was considered statistically significant.

\section{RESULTS}

Among 52 patients who were enrolled in this study, there were 36 males (69\%) and 16 females (31\%). The mean age of all patients studied was 59.2 years $(+58.6)$. The mean of preoperative NLR in these 52 patients was $6.35(+4.01)$, with a range of 1.48-21.0. The clinical characteristic of patients can be seen in Table 1.

Table 1. Clinical characteristic of the study population

\begin{tabular}{|c|c|c|}
\hline Characteristics & $\begin{array}{l}\text { Total } \\
\text { (n (\%)) }\end{array}$ & $\mathbf{p}$ \\
\hline Age (mean; range) & $59.2(31-72)$ & N/A \\
\hline $\begin{array}{l}\text { Gender } \\
\quad \text { Male } \\
\text { Female } \\
\text { Male-female ratio }\end{array}$ & $\begin{array}{l}36(69) \\
16(31) \\
7: 3\end{array}$ & $<.0001$ \\
\hline $\begin{array}{l}\text { Preoperative } \\
\text { neutrophil-lymphocyte } \\
\text { ratio (mean; range) }\end{array}$ & $6.35(1.48-21.0)$ & \\
\hline $\begin{array}{l}\text { Tumor staging } \\
\text { T1 } \\
\text { T2 } \\
\text { T3 } \\
\text { T4 }\end{array}$ & $\begin{array}{l}0(0) \\
20(38) \\
9(17) \\
23(44)\end{array}$ & $<.0001$ \\
\hline $\begin{array}{l}\text { Fuhrman grade } \\
\qquad \begin{array}{l}1 \\
2 \\
3 \\
4\end{array}\end{array}$ & $\begin{array}{l}0(0) \\
18(34) \\
24(46) \\
10(19)\end{array}$ & $<.0001$ \\
\hline $\begin{array}{l}\text { Length of stay* (day) } \\
\text { (mean; range) } \\
\text { Low NLR group } \\
\text { High NLR group }\end{array}$ & $\begin{array}{ll}18 & (12-22) \\
25 & (16-30)\end{array}$ & $<.0001$ \\
\hline $\begin{array}{l}\text { Mortality } \\
\text { Died } \\
\text { Alive }\end{array}$ & $\begin{array}{ll}29 & (55.8) \\
23 & (44.2)\end{array}$ & $<.0001$ \\
\hline $\begin{array}{l}\text { Recurrence } \\
\text { Yes } \\
\text { No }\end{array}$ & $\begin{array}{l}3(5.8) \\
49(94.2)\end{array}$ & $<.0001$ \\
\hline
\end{tabular}

Intergroup differences of gender, mortality, and recurrence were analyzed using two-sample t-test, while tumor staging and Fuhrman grade were analyzed using one-way analysis of variance.

*The cut-off point of NLR was determined as 3.01 
Statistically, the NLR value was significantly associated with tumor pathologic stage $(p=0.020)$, length of stay of the patients $(p=0.00)$, and the mortality rate $(p=$ 0.012). Neutrophil lymphocyte ratio was not statistically significant with Fuhrman tumor grade $(p=0.130)$, and tumor recurrence $(p=0.670)$.

In this study, the number of samples was more than 50 subjects, so the normality test was chosen using the Kolmogorov-Smirnov analytical test. The distribution of NLR was normal, so a parametric analytical test was performed using two-sample t-test. The analytic test can be seen in Table 2 .

Table 2. The results of comparative and correlative test

\begin{tabular}{lll}
\hline $\begin{array}{l}\text { Preoperative neutrophil-lymphocyte } \\
\text { ratio }(\mathbf{n}=\mathbf{5 2})\end{array}$ & $\mathbf{p}$ & $\mathbf{r}$ \\
\hline Staging & 0.020 & 0.322 \\
Fuhrman Grade & 0.357 & 0.130 \\
Length of Stay & 0.000 & 0.527 \\
Mortality & 0.012 & 0.345 \\
Recurrence & 0.670 & 0.06 \\
\hline
\end{tabular}

Univariate analysis of factors associated with NLR was examined by Pearson's correlation analysis

In the analysis test carried out in this study, the value of NLR was significantly associated with tumor pathologic stage $(p=0.020)$ with weak correlation strength $(r=0.322)$. The value of NLR was associated with the length of stay of the patients $(p=0.00)$ with strong correlation strength $(r=0.527)$. The value of NLR was associated with the mortality rate $(p=0.012)$ with weak correlation strength $(r=0.345)$. Neutrophil lymphocyte ratio was not statistically significant with Fuhrman tumor grade $(p=0.357)$ and tumor recurrence $(p=0.670)$.

\section{DISCUSSION}

To date, kidney cancer is still one of the most common cancers worldwide with high morbidity and mortality [8]. Renal cell carcinoma (RCC) is the most common type of malignancy [8]. Several predictor factors have been extensively studied to estimate the clinical significance in kidney tumor patients [9]. We used the neutrophil-lymphocyte ratio as a predictor factor in kidney cancer as this variable is considered an easy and economical biomarker to predict systemic inflammation process as implicated in cancer development and progression. The present study demonstrated that preoperation levels of NLR that represent systemic inflammatory response were significantly associated with tumor staging, length of stay, and mortality in patients undergoing surgical resection.

Cancer and inflammation are associated with increasing evidence that tumor-associated inflammation and tumor microenvironment play a more and more important role in cancer development, progression, and metastasis and might be related to systemic inflammation $[10,11]$. Inflammatory responses in the tumor microenvironment have been reflected by some common markers in peripheral blood, for example, some cytokines, leucocytes, and their subtypes [12]. However, further studies investigating the prognostic impact of the peripheral blood component in localized RCC patients are warranted [13].

A high NLR reflects both an increased neutrophildependent inflammatory reaction and a decreased lymphocyte-mediated antitumor response immunity [14]. Previous studies mentioned these factors may have an association with aggressive tumor biology, cancer progression, and poor prognosis [15]. Neutrophils exhibit a significant role in the inflammation process within the tumor. For instance, this is shown by the cytokines tumor necrosis factor, interleukin (IL)-1, and IL-6, and the pro-angiogenic vascular endothelial growth factor (VEGF) produced by circulated neutrophil [16]. In addition, a lower count of CD4+ T-helper lymphocytes may be shown in a relative lymphocytopenia condition, causing poor lymphocyte-mediated immunity against cancer [17]. Therefore, a combination of these two variables, which is calculated as NLR, may provide further prognostic knowledge in malignancy and strongly predict the outcome [18].

There is increasing awareness of the importance of lymphocytes for human adaptive immunity, particularly the tumor-infiltrating lymphocytes (TILs) in various immune pathways in the tumor microenvironment [19]. Lower lymphocyte count may indicate a suboptimal immune response against malignancy and result in a bad prognosis [20]. The CD8+T cells contributed prominently to restrict tumor growth by inducing apoptosis of the cancer cells and cytotoxic activity pathways [21]. Meanwhile, CD4+T cells are an important source of IL-2, which is critical for CD8+T cell development [22]. In addition, the current study mentioned that CD4+ T cells support the polyfunctionality of cytotoxic CD8+ $T$ cells with its memory potential in immunological control of cancer [23]. On the other hand, neutrophils may inhibit the immune response by suppressing the cytolytic action of lymphocytes, natural killer (NK) cells, and activated T cells [24]. This is supported by evidence that low lymphocyte count was associated with poor outcomes in advanced cancer patients [25], while increased TILs were correlated with a better prognosis, suggesting an effective antitumor immune response [24]. As a result, several studies have 
shown that elevated NLR was associated with poor survival in various malignancies. Our results also indicated that preoperative elevated NLR was significantly related to mortality.

Our findings also suggested the correlation of preoperative NLR with tumor staging. This is in concordance with previous studies, proposing a positive correlation between NLR levels and T staging [26,27].

To the best of our knowledge, this study is one of the first in any discipline to discuss the correlation between hospital stay and the preoperative NLR level in RCC patients, with no previous studies that specifically investigated it. Nevertheless, it is imperative to consider the strong correlation between the NLR level and the immunity status of a patient with malignancy [14], which might be affecting the recovery process and thus prolonged the hospital stay. However, this still warrants further investigation.

We also conclude that there is not a significant linear correlation between NLR levels with Fuhrman grade and recurrence rate in our study population. These findings are different from those of the previous study, mostly suggesting that the elevation of NLR will also increase the Fuhrman grade and vice versa $[28,29]$. For recurrence rate, previous studies found that high NLR was correlated with a high tendency of recurrence rate $[30,31]$. Further studies are needed to confirm these differences.

We are aware of several limitations of the present study. First, this study has retrospective designs. Second, it was conducted at a single center with a small number of subjects. Multicenter, cross-country prospective studies involving a larger sample of RCC patients are needed to confirm our findings. Third, we did not take into account the risk stratification based on other RCC risk factors such as family history, smoking habits, hypertension, and excess body weight in this study, which could influence the prognosis. We believe that this study has important clinical implications and can form the basis of science in predicting the prognosis of patients who have kidney tumor disease.

\section{CONCLUSIONS}

The NLR role to predict the prognosis of RCC patients in the future has to be considered as a valid approach. The NLR has a positive correlation for several variables of the RCC population in our center, but to determine the prime role of NLR and what type of prognosis this parameter has in RCC, further survival analysis is warranted. Although further studies with larger samples need to be performed, these easily measurable biomarkers showed a promising result, especially to predict tumor stage, length of hospital stay, and mortality rate.

\section{DECLARATIONS}

\section{Ethics approval and consent to participate}

The study was held after approval from the Ethics Committee, Faculty of Medicine, Public Health and Nursing Universitas Gadjah Mada (study number KE/ FK/1060/EC/2020).

\section{Competing interest}

The authors declare no competing interest in this study

\section{Acknowledgment}

The authors would like to thank our supervisor Dr. dr Danarto, Sp.B, Sp.U(K) for his guidance, great support, and kind advice throughout this research studies.

\section{REFERENCES}

1. Siegel RL, Miller KD, Jemal A. Cancer statistics, 2015. CA. Cancer J Clin. 2015;65(1):5-29.

2. Siegel RL, Miller KD, Jemal A. Cancer statistics, 2018. CA. Cancer J Clin. 2018;68(1):7-30.

3. Padala SA, Barsouk A, Thandra KC et al. Epidemiology of renal cell carcinoma. Onkologe. 2019;11(3):79-87.

4. Volpe A, Patard JJ. Prognostic factors in renal cell carcinoma. World J Urol. 2010;28(3):319-27.

5. Lee JS, Kim NY, Na SH, et al. Reference values of neutrophil-lymphocyte ratio, lymphocyte-monocyte ratio, platelet-lymphocyte ratio, and mean platelet volume in healthy adults in South Korea. Medicine. 2018;97(26):e11138.

6. Rajandram R, Perumal K, Yap NY. Prognostic biomarkers in renal cell carcinoma: is there a relationship with obesity? Transl Androl Urol. 2019;8(Suppl 2):S138-S46.

7. Paner GP, Stadler WM, Hansel DE, et al. Updates in the eighth edition of the tumor-node-metastasis staging classification for urologic cancers. Eur Urol. 2018;73(4):560-9.

8. James SL, Abate D, Abate $\mathrm{KH}$, et al. Global, regional, and national incidence, prevalence, and years lived with disability for 354 Diseases and Injuries for 195 countries and territories, 1990-2017: A systematic analysis for the Global Burden of Disease Study 2017. Lancet. 2018;392(10159):1789-858.

9. Klatte T, Rossi SH, Stewart GD. Prognostic factors and prognostic models for renal cell carcinoma: A literature review. World J Urol. 2018;36(12):1943-52.

10. Galdiero MR, Bonavita E, Barajon I, et al. Tumor associated macrophages and neutrophils in cancer. Immunobiology. 2013;218(11):1402-10.

11. Van Verschuer VMT, Hooning MJ, Van BaareGeorgieva RD, et al. Tumor-associated inflammation as a potential prognostic tool in BRCA1/2-associated breast cancer. Hum Pathol. 2015;46(2):182-190. 
12. Mei Z, Shi L, Wang B, et al. Prognostic role of pretreatment blood neutrophil-to-lymphocyte ratio in advanced cancer survivors: A systematic review and meta-analysis of 66 cohort studies. Cancer Treat Rev. 2017;58:1-13.

13. Otunctemur A, Dursun $M$, Besiroglu $\mathrm{H}$ et al. Clinical significance of preoperative neutrophil - to lymphocyte ratio in renal cell carcinoma. Int Braz J Urol. 2016;42(4):678-84.

14. Minardi D, Scartozzi M, Montesi L, et al. Neutrophilto-lymphocyte ratio may be associated with the outcome in patients with prostate cancer. Springerplus. 2015;4(1):1-5.

15. Bolat D, Aydoğdu Ö, Polat $S$, et al. Predictive value of preoperative neutrophil-to-lymphocyte ratio on the prognosis of germ cell testicular tumors. Turk Urol Derg. 2017;43(1):55-61.

16. Kany S, Vollrath JT, Relja B. Cytokines in inflammatory disease. Int J Mol Sci. 2019;20(23):1-31.

17. Vijayakumar S, Viswanathan S, Aghoram R. Idiopathic CD4 lymphocytopenia : Current insights. Immunotargets Ther. 2020;9:79-93.

18. Zhang $X$, Zhang W, Feng LJ. Prognostic significance of neutrophil lymphocyte ratio in patients with gastric cancer: A meta-analysis. PLoS One. 2014;9(11):e111906.

19. Binnewies $M$, Roberts EW, Kersten $K$, et al. Understanding the tumor immune microenvironment (TIME) for effective therapy. Nat Med. 2018;24(5):541-50.

20. Dai $T$, Lin $G$, Deng $M$, et al. The prognostic significance of neutrophil-to-lymphocyte ratio at different time points in patients with hepatocellular carcinoma receiving liver resection. Transl Cancer Res. 2020;9(2):441-57.

21. Pluhar GE, Pennell CA, Olin MR. CD8 + T Cell Independent immune-mediated mechanisms of antitumor activity. 2015;35(2):153-72.

22. Han X, Bai S, Cui Y, et al. Essential role of CD4+ T cells for the activation of group 2 innate lymphoid cells during respiratory syncytial virus infection in mice. Immunotherapy. 2019;11(15):1303-13.
23. Imai N, Tawara I, Yamane $\mathrm{M}$, et al. CD4+ $\mathrm{T}$ cells support polyfunctionality of cytotoxic CD8+ T cells with memory potential in immunological control of tumor. Cancer Sci. 2020;111(6):1958-68.

24. Yamanaka T, Matsumoto S, Teramukai, $S$ et al. The baseline ratio of neutrophils to lymphocytes is associated with patient prognosis in advanced gastric cancer. Oncology. 2008; 73(3-4):215-20.

25. Fogar P, Sperti C, Basso D, et al. Decreased total lymphocyte counts in pancreatic cancer: An index of adverse outcome. Pancreas. 2006;32(1):22-8.

26. Özgehan G, Kahramanca Ş, Kaya IO, et al. Neutrophillymphocyte ratio as a predictive factor for tumor staging in colorectal cancer. Turkish J Med Sci. 2014;44(3):365-8.

27. Tan YG, Sia J, Huang HH, Lau WKO. Neutrophil-tolymphocyte ratio independently predicts advanced pathological staging and poorer survival outcomes in testicular cancer. Investig Clin Urol. 2019;60(3):17683.

28. Kisa E, Yucel C, Keskin MZ, et al. The role of hematological parameters in predicting fuhrman grade and tumor stage in renal cell carcinoma patients undergoing nephrectomy. Medicina. 2019;55(6):287.

29. Arda E, Yuksel I, Cakiroglu B, et al. Valuation of Neutrophil/Lymphocyte Ratio in Renal Cell Carcinoma Grading and Progression. Cureus. 2018;10(1):e2051.

30. Patel A, Ravaud A, Motzer RJ, et al. Neutrophil-tolymphocyte ratio as a prognostic factor of diseasefree survival in postnephrectomy high-risk locoregional renal cell carcinoma: Analysis of the S-TRAC trial. Clin Cancer Res. 2020;26(18):4863- 68.

31. Lalani AA, Xie W, Martini DJ, et al. Change in neutrophil-to-lymphocyte ratio (NLR) in response to immune checkpoint blockade for metastatic renal cell carcinoma. J Immunother. Cancer. 2018;6(1):5. 